Proyecciones

Vol. 28, No 1, pp. 57-73, May 2009.

Universidad Católica del Norte

Antofagasta - Chile

\title{
A NOTE ON THE FIBER DIMENSION THEOREM
}

\author{
JACQUELINE ROJAS * \\ $U F P B, B R A S I L$ \\ and \\ RAMÓN MENDOZA \\ UFPE, BRASIL
}

Received : February 2009. Accepted : April 2009

\begin{abstract}
The aim of this work is to prove a version of the Fiber Dimension Theorem, emphasizing the case of non-closed points.

Resumo

El objetivo de este trabajo es probar una versión del Teorema de la Dimensión de la Fibra, enfatizando el caso de puntos no cerrados.
\end{abstract}

MSC 2000: $14 A 15$

Key words: Fiber dimension theorem, non-closed points

${ }^{*}$ Partially supported by PADCT - CNPq 


\section{INTRODUCTION}

The fiber dimension theorem is an important mathematical tool in the everyday life of an algebraic geometer. For example, we can calculate the dimension of the space of matrices whose rank is less than or equal to $k$; the dimension of the incidence variety $\Gamma=\{(\pi, \ell, p) \mid p \in \ell \subset \pi\}$ where $\pi$ is a plane, $\ell$ is a line and $p$ is a point in some projective space as indicated in the figure below (see Harris [8] for other applications).

We can also use this theorem to know for example if a nonsingular hypersurface contains a finite number of lines, planes, etc., in particular, we can show that a nonsingular cubic surface in the three-dimensional projective space contains exactly 27 lines (see Reid [12], Beauville [3], CrauderMiranda [4]).

The aim of this article is to prove the following theorem.

1.1. Theorem. (Fiber dimension theorem) Let $X$ and $Y$ be integral affine schemes of finite type over a field $\mathbf{K}, f: X \longrightarrow Y$ be a dominant morphism. Then there exists a nonempty open subset $U \subseteq Y$ such that $\operatorname{dim} f^{-1}(\mathrm{y})=r$ for all $\mathrm{y} \in U(r=\operatorname{dim} X-\operatorname{dim} Y)$.

Indeed, the theorem above can be read from Eisenbud's compendium, Corollary 14.5 (see p. 310 in [6]). But, the strategy that we will use to prove the theorem 1.1 above, was suggested by exercise 3.22 in Hartshorne's book [10] at page 95. In fact, the exercise 3.22 in Hartshorne's book tell us that it is enough to prove the following theorem.

1.2. Theorem. (see Theorem 3 in chapter I, section 8 of [11]) Let $X$ and $Y$ be affine schemes and $f: X \longrightarrow Y$ be a dominant morphism. Let $r=\operatorname{dim} X-\operatorname{dim} Y$, then there exists $U$ a nonempty open subset of $Y$ such that

1. $f(X) \subset U$. 
2. If $W$ is any irreducible closed subset of $Y$ such that $W \cap U \neq \emptyset$ and $Z$ is an irreducible component of $f^{-1}(W)$ such that $Z \cap f^{-1}(U) \neq \emptyset$ then we have that $\operatorname{dim} Z=\operatorname{dim} W+r$.

It is important to notice that the proof of Theorem 1.1 is not just an application of Theorem 1.2, due to the existence of non-closed points, as is the case of affine schemes with the Zariski topology (see Section 3). In fact, in this work we are not going to prove Theorem 1.2. We focus our attention to the proofs of the results that we will use to conclude Theorem 1.1 in the case of non-closed points, showing that, we need to be more careful with these ones.

For those who are not familiar with the nature of algebraic varieties, in particular with schemes of points, where we can find non-closed points, fat points, etc., we recommend the beautiful exposition in Harris-Eisenbud [9], where certain fat points appear as the limits of a finite number of simple points and other very interesting results. Also in [7] you can find some results on fat points in the projective plane made by Harbourne.

We refer the reader to the study of quadruplets of points in the projective plane made by Avritzer-Vainsencher in [2] and conical sextuplets made by Rojas-Vainsencher in [13], where they give an explicit descriptions of the varieties that parametrize these subschemes of degree 4 (respectively 6) contained in a conic.

\section{Basic definitions and examples}

\subsection{Affine varieties and Zariski's topology}

By the affine space over a field $\mathbf{C}$, we mean simply the vector space $\mathbf{C}^{n}$; this is usually denoted by $\mathbf{A}^{n}$ (the main distinction between affine space and the vector space $\mathbf{C}^{n}$ is that the origin plays no special role in affine space). An affine variety $\mathbf{X}$ in $\mathbf{A}^{n}$ is simply the common zero locus of a collection of polynomials in $\mathbf{C}\left[x_{1}, \ldots, x_{n}\right]$.

More precisely, let $f_{1}, \ldots, f_{k} \in \mathbf{C}\left[x_{1}, \ldots, x_{n}\right]$ be polynomials. Then

$$
Z\left(\left\{f_{1}, \ldots, f_{k}\right\}\right)=\left\{\left(a_{1}, \ldots, a_{n}\right) \in \mathbf{C}^{n} \mid f_{i}\left(a_{1}, \ldots, a_{n}\right)=0 \forall i=1, \ldots, k\right\},
$$

is the affine variety in $\mathbf{A}^{n}$ determined by the polynomials $f_{1}, \ldots, f_{k}$. 
Thus, if we consider $f=0 \in \mathbf{C}\left[x_{1}, \ldots, x_{n}\right]$ then we have that $\mathbf{A}^{n}=Z(0)=\mathbf{C}^{n}$.

\subsection{Remarks.}

1. Let $I \subseteq \mathbf{C}\left[x_{1}, \ldots, x_{n}\right]$ be an ideal. Assume that $I$ is generated by $f_{1}, \ldots, f_{k} \in \mathbf{C}\left[x_{1}, \ldots, x_{n}\right]$ then we define $Z(I)=Z\left(\left\{f_{1}, \ldots, f_{k}\right\}\right)$ as in (2.1). Note that $Z(I)$ is independent of the choice of the generators of $I$.

2. Let $S$ be any collection of polynomials in $\mathbf{C}\left[x_{1}, \ldots, x_{n}\right]$. Then we put $Z(S)=Z(\langle S\rangle)$ where $\langle S\rangle$ is the ideal in $\mathbf{C}\left[x_{1}, \ldots, x_{n}\right]$ generated by $S$.

2.2. Examples. Let us consider the ring $\mathbf{C}\left[x_{1}, x_{2}\right]$.

1. $Z(\{1\})=\emptyset$.

2. $Z(\{0\})=\mathbf{A}^{2}$, it is called affine plane.

3. $Z\left(\left\langle x_{2}\right\rangle\right)=\left\{(a, 0) \in \mathbf{C}^{2}\right\}$ is an affine line.

The Zariski Topology on $\mathbf{A}^{n}$, is simply the topology where the closed sets are given by $Z(I)$ for some ideal $I \subseteq \mathbf{C}\left[x_{1}, \ldots, x_{n}\right]$. Let us see some examples.

\subsection{Examples.}

1. Every point $\left(a_{1}, \ldots, a_{n}\right)$ in $\mathbf{A}^{n}$ is closed. In fact, $\left\{\left(a_{1}, \ldots, a_{n}\right)\right\}=$ $Z\left(x_{1}-a_{1}, \ldots, x_{n}-a_{n}\right)$.

2.

The set $X=\{(a, 0) \mid a \in \mathbf{C}\}^{c} \cup\{(0,0)\}=$

is neither closed nor open. Whereas $U=X-\{(0,0)\}$ is open, since 


\section{Affine schemes and non-closed points}

\subsection{The Zariski topology on $\operatorname{Spec}(A)$}

Let $A$ be a commutative ring with unity and

$$
\operatorname{Spec}(A)=\{\mathrm{x} \subset A \mid \mathrm{x} \text { is a prime ideal of } A\},
$$

be the prime spectrum of $A$.

\subsection{Remarks.}

1. If the ring $A$ is an integral domain then we will denote by $0 \in$ $\operatorname{Spec}(A)$ the prime ideal $\langle 0\rangle$, where $0 \in A$ is the zero element of the ring $A$.

2. We will use the notation $\mathbf{A}_{\mathbf{C}}^{n}$ for $\operatorname{Spec}\left(\mathbf{C}\left[x_{1}, \ldots, x_{n}\right]\right)$.

Note that, $\mathbf{A}^{n}$ can be considered as a subset of $\mathbf{A}_{\mathbf{C}}^{n}=\operatorname{Spec}\left(\mathbf{C}\left[x_{1}, \ldots, x_{n}\right]\right)$ through the following correspondence

$$
\left(a_{1}, \ldots, a_{n}\right) \longmapsto\left\langle x_{1}-a_{1}, \ldots, x_{n}-a_{n}\right\rangle
$$

where to each point $\left(a_{1}, \ldots, a_{n}\right) \in \mathbf{A}^{n}$ we associate the maximal ideal $\left\langle x_{1}-a_{1}, \ldots, x_{n}-a_{n}\right\rangle$ in $\mathbf{C}\left[x_{1}, \ldots, x_{n}\right]$.

Thus the above identification implies that $\mathbf{A}_{\mathbf{C}}^{1}=\operatorname{Spec}(\mathbf{C}[x])$ possesses exactly one more point than $\mathbf{A}^{1}$, namely $\mathbf{x}=0$. Nevertheless, in the case $n>1$, there are an infinite number of points, or more precisely, of prime ideals.

Then we can make the following question:

Is it possible to define a topology in $\mathbf{A}_{\mathbf{C}}^{n}=\operatorname{Spec}\left(\mathbf{C}\left[x_{1}, \ldots, x_{n}\right]\right)$ such that under the identification in (3.2) the points of $\mathbf{A}^{n}$ still remain closed?

Yes, it is enough to observe that for any commutative ring with unity $A$, the family of sets $V(I)=\{\mathrm{x} \in \operatorname{Spec}(A) \mid I \subseteq \mathrm{x}\}$ with $I \subseteq A$ an ideal of $A$, defines a topology on $\operatorname{Spec}(A)$, called the Zariski topology of $\operatorname{Spec}(A)$ (see problem 15, p. 12 in Atiyah-MacDonald [1]). 
In fact, we have the following bijection

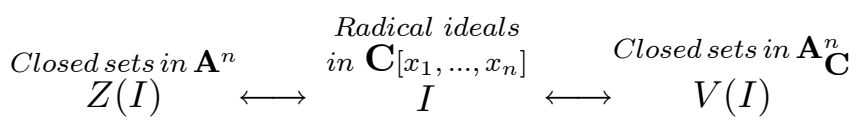

(see p. 4 in Hartshorne [10])

Coming back to the case of $\mathbf{A}_{\mathbf{C}}^{1}$, we would like to known: is $\mathrm{x}=0$ a closed point of $\mathbf{A}_{\mathbf{C}}^{1}$ or not?

Note that, $\overline{\{0\}} \subset V(0)$ since $0 \in V(0)$. On the other hand

$$
\overline{\{0\}}=\bigcap_{0 \in V(I)} V(I) \text {. }
$$

Therefore, if $0 \in V(I)$ then we have that $I \subseteq 0$, that is, $V(I)=\operatorname{Spec}(\mathbf{C}[x])=$ $\mathbf{A}_{\mathbf{C}}^{1}$. From this we conclude that $\overline{\{0\}}=V(0)=\operatorname{Spec}(\mathbf{C}[x])=\mathbf{A}_{\mathbf{C}}^{1}$. Thus, $\mathrm{x}=0$ is not a closed point in $\mathbf{A}_{\mathbf{C}}^{1}$. In fact, 0 is the generic point of $\mathbf{A}_{\mathbf{C}}^{1}$. More generally, if $X$ is a topological space and $\mathrm{x} \in X$ is a point of $X$ such that $\overline{\{\mathrm{x}\}}=X$, then $\mathrm{x}$ is called a generic point of $X$ (see p. 74 in Hartshorne $[10])$.

\subsection{Remarks.}

1. The consequence of 0 to be the generic point of $\mathbf{A}_{\mathbf{C}}^{1}$ follows from the fact that $\mathbf{C}[x]$ is an integral domain, as the following result guarantees.

Let $A$ be an integral domain then $0 \in \operatorname{Spec}(A)$ is the generic point of $\operatorname{Spec}(A)$.

2. Using a similar reasoning for $\mathrm{x} \in \operatorname{Spec}(A)$ in place of $0 \in$ $\operatorname{Spec}(\mathbf{C}[x])$ above, we conclude that $\overline{\{\mathrm{x}\}}=V(\mathrm{x})$. From this, we can deduce that,

$\mathrm{x}$ is a generic point of $\operatorname{Spec}(A)$ if and only if $\mathrm{x}=N_{A}$. $N_{A}$ denote the nilradical of the $\operatorname{ring} A$ as in (4.1).

\subsection{The sheaf of regular functions on $\operatorname{Spec}(A)$}

Let $X$ be any topological space. A presheaf $\mathcal{F}$ on $X$ assigns to each open set $U$ in $X$ a set, denoted $\mathcal{F}(U)$, and to every pair of nested open sets $U \subset V \subset X$ a restriction map

$$
r_{V, U}: \mathcal{F}(V) \longrightarrow \mathcal{F}(U)
$$


satisfying the basic property that

$$
\begin{aligned}
r_{U, U}=i d_{U} & \forall U \subset X, \\
r_{V, U} \circ r_{W, V}=r_{W, U} & \forall U \subset V \subset W \subset X .
\end{aligned}
$$

A presheaf $\mathcal{F}$ on $X$ is a sheaf if for each open covering $U=\cup_{a \in I} U_{a}$ of an open set $U \subset X$ and each collection of elements $f_{a} \in \mathcal{F}\left(U_{a}\right)$ for each $a \in I$ having the property that

$$
r_{U_{a}, U_{a} \cap U_{b}}\left(f_{a}\right)=r_{U_{b}, U_{a} \cap U_{b}}\left(f_{b}\right) \quad \forall a, b \in I
$$

there is a unique element $f \in \mathcal{F}(U)$ such that $r_{U, U_{a}}(f)=f_{a} \forall a \in I$.

3.3. Remark. If each $\mathcal{F}(U)$ is a group, ring, etc. and each restriction map $r_{V, U}$ is an homomorphism of groups, rings, etc. then $\mathcal{F}$ is called a presheaf of groups, rings, etc.

The concept of $\mathcal{B}$-sheaf (which will be soon introduced) will allow us to define a sheaf from certain special types of bases for the topological space $X$. Thus it is not necessary to define a sheaf in all the open sets of the topology of $X$.

Given a base $\mathcal{B}$ for the open sets of a topological space $X$, we say that a collection of sets, groups, rings, etc. $\mathcal{F}(U)$ for open sets $U \in \mathcal{B}$ and maps $r_{V, U}: \mathcal{F}(V) \longrightarrow \mathcal{F}(U)$ for $V \subset U$ form a $\mathcal{B}$-sheaf if they satisfy the sheaf axiom with respect to inclusions of basic open sets in basic open sets and coverings of basic open sets by basic open sets. (The condition that $f_{a} \in \mathcal{F}\left(U_{a}\right)$ for each $a \in I, U_{a} \in \mathcal{B}$ having the property $r_{U_{a}, U_{a} \cap U_{b}}\left(f_{a}\right)=r_{U_{b}, U_{a} \cap U_{b}}\left(f_{b}\right) \forall a, b \in I ; U_{a}, U_{b} \in \mathcal{B}$ must be replaced by the following condition

$$
\left.r_{U_{a}, V}\left(f_{a}\right)=r_{U_{b}, V}\left(f_{b}\right) \quad \forall a, b \in I ; U_{a}, U_{b}, V \in \mathcal{B} \text { such that } V \subset U_{a} \cap U_{b} .\right)
$$

And it can be shown that.

3.4. Proposition. Every $\mathcal{B}$-sheaf on $X$ extends uniquely to a sheaf on $X$.

Proof. See p. 17 in Harris-Eisenbud [9]. 
Coming back to the topological space $\operatorname{Spec}(A)$ defined in (3.1) we have that

$$
\operatorname{Spec}(A)_{f}=\operatorname{Spec}(A)-V(f)=\{\mathrm{x} \in \operatorname{Spec}(A) \mid f \notin \mathrm{x}\} \quad \text { with } f \in A,
$$

form a base for the Zariski topology on $\operatorname{Spec}(A)$. The open sets $\operatorname{Spec}(A)_{f}$ are called distinguished (or basic) open sets of $\operatorname{Spec}(A)$.

We define the sheaf of regular functions $\mathcal{O}_{\operatorname{Spec}(A)}$ on $\operatorname{Spec}(A)$ as follows

$$
\mathcal{O}_{\operatorname{Spec}(A)}\left(\operatorname{Spec}(A)_{f}\right):=A_{f} \quad \forall f \in A
$$

where $A_{f}$ denoted the localization of the ring $A$ at $f \in A$.

Note that, $\operatorname{Spec}(A)_{g} \subset \operatorname{Spec}(A)_{f}$ if and only if some power of $g$ is a multiple of $f$ then we define the restriction map

$$
\begin{aligned}
r_{\operatorname{Spec}(A)_{f}, \operatorname{Spec}(A)_{g}:} A_{f} & \longrightarrow A_{g} \\
\frac{a}{f^{k}} & \longmapsto \frac{a \mu^{k}}{g^{N k}} \quad \text { where } g^{N}=\mu f .
\end{aligned}
$$

In fact, if $\mathcal{B}$ is the collection of distinguished open sets $\operatorname{Spec}(A)_{f}$ of $\operatorname{Spec}(A)$ then $\mathcal{O}_{\text {Spec }(A)}$ is a $\mathcal{B}$-sheaf on $\operatorname{Spec}(A)$ (see proposition I-18, p. 19 in HarrisEisenbud [9]).

\section{Affine schemes}

An affine scheme is a pair $\left(\operatorname{Spec}(A), \mathcal{O}_{\operatorname{Spec}(A)}\right)$ where $\operatorname{Spec}(A)$ is the prime spectrum of the commutative ring $A$ considered as a topological space with the Zariski topology and $\mathcal{O}_{\operatorname{Spec}(A)}$ is the sheaf of regular functions over $\operatorname{Spec}(A)$.

\subsection{Remarks.}

1. Let $\left(\operatorname{Spec}(A), \mathcal{O}_{\operatorname{Spec}(A)}\right)$ be an affine scheme, then the ring $A$ is called the coordinate ring of this affine scheme.

2. The closed set $V(I) \subset \mathbf{A}_{\mathbf{C}}^{n}$ can be identified with the affine scheme

$$
\left(\operatorname{Spec}\left(\mathbf{C}\left[x_{1}, \ldots, x_{n}\right] / \sqrt{I}\right), \mathcal{O}_{\operatorname{Spec}\left(\mathbf{C}\left[x_{1}, \ldots, x_{n}\right] / \sqrt{I}\right)}\right),
$$

where $\sqrt{I}=\left\{p \in \mathbf{C}\left[x_{1}, \ldots, x_{n}\right] \mid p^{n} \in I\right.$ for some $\left.n \in \mathbf{N}\right\}$ is called the radical of $I$. An ideal $I$ in the $\operatorname{ring} A$ is called radical if $\sqrt{I}=I$.

In the next section, we will define morphisms between affine schemes. 


\subsection{Morphisms between affine schemes}

Let us take $X=\operatorname{Spec}(A)$ and $Y=\operatorname{Spec}(B)$ with $A$ and $B$ commutative rings with unity. Note that every homomorphism of rings $\varphi: B \longrightarrow A$ determines a continuous function $\varphi^{*}: X \longrightarrow Y$, defined by $\varphi^{*}(\mathrm{x})=\varphi^{-1}(\mathrm{x})$.

We will say that $f: X \longrightarrow Y$ is a morphism between affine schemes if it is obtained from a ring homomorphism, that is, there exist $\varphi: B \longrightarrow A$ us above such that $f=\varphi^{*}$.

4.2. Remark. More precisely a morphism between two affine schemes is a pair $\left(f, f^{\#}\right)$, where $f: \operatorname{Spec}(A) \longrightarrow \operatorname{Spec}(B)$ is continuous function and $f^{\#}: \mathcal{O}_{\operatorname{Spec}(B)} \longrightarrow f_{*} \mathcal{O}_{\text {Spec }(A)}\left(f_{*} \mathcal{O}_{\text {Spec }(A)}\right.$ is a sheaf over $\operatorname{Spec}(B)$ defined by $f_{*} \mathcal{O}_{\operatorname{Spec}(A)}(V)=\mathcal{O}_{\operatorname{Spec}(A)}\left(f^{-1}(V)\right)$ for $V \subseteq \operatorname{Spec}(B)$ open and it is called the direct image of $\mathcal{O}_{\operatorname{Spec}(A)}$ by $f$ ), is a family of ring homomorphisms $\left\{f_{\text {Spec }(B)_{g}}^{\#}\right\}_{g \in B}$ commuting with the restriction maps given by

$$
\begin{aligned}
f^{\#}{ }_{\operatorname{Spec}(B)_{g}}: \mathcal{O}_{\operatorname{Spec}(B)}\left(\operatorname{Spec}(B)_{g}\right)=B_{g} & \longrightarrow f_{*} \mathcal{O}_{\operatorname{Spec}(A)}\left(\operatorname{Spec}(B)_{g}\right)=A_{\varphi(g)} \\
\frac{b}{g^{k}} & \longmapsto \frac{\varphi(b)}{\varphi(g)^{k}}
\end{aligned}
$$

where we assume that $f=\varphi^{*}$ for some ring homomorphism $\varphi: B \longrightarrow A$ and we use the basic fact that $f^{-1}\left(\operatorname{Spec}(B)_{g}\right)=\operatorname{Spec}(A)_{\varphi(g)}$ for all $g \in B$.

In fact, we have the following equivalence of categories

$$
\begin{array}{ccc}
\{\text { Rings }\} & \longrightarrow & \{\text { Affine schemes }\} \\
B & \longmapsto & \left(\operatorname{Spec}(B), \mathcal{O}_{\operatorname{Spec}(B)}\right) \\
\downarrow \varphi & \uparrow\left(\varphi^{*}, \varphi^{\#}\right) \\
A & \longmapsto & \left(\operatorname{Spec}(A), \mathcal{O}_{\operatorname{Spec}(A)}\right)
\end{array}
$$

Thus we have that $\operatorname{Hom}_{\text {Rings }}(B, A) \cong \operatorname{Hom}_{\text {AffineSchemes }}(X, Y)$. That is, each morphism between affine schemes is determined by a unique homomorphism of rings (see Theorem I-40 in Harris-Eisenbud [9]).

Let us see some examples.

\subsection{Examples.}

1. Let $A$ be a ring, $I \subset A$ be an ideal and $\pi: A \longrightarrow A / I$ the canonical homomorphism. Then $\pi$ induces the morphism

$$
f: V(I) \longrightarrow \operatorname{Spec}(A) .
$$


2. There exists a morphism $f: \mathbf{A}_{\mathbf{C}}^{2} \longrightarrow \mathbf{A}_{\mathbf{C}}^{1}$ such that $f(a, b)=a$ on the closed points of $\mathbf{A}^{2}$ ?

The answer will be affirmative if we can find $\varphi: \mathbf{C}[t] \longrightarrow$ $\mathbf{C}[u, v]$ such that $\varphi^{-1}\langle u-a, v-b\rangle=\langle t-a\rangle$.

We can define $\varphi$ by $\varphi(t)=u$. Then, since $\langle u-a, v-b\rangle$ is a prime ideal of $\mathbf{C}[u, v]$, we have that $\varphi^{-1}\langle u-a, v-b\rangle$ is also a prime ideal of $\mathbf{C}[t]$. Note that $\varphi(t-a)=u-a$, then $t-a \in$ $\varphi^{-1}\langle u-a, v-b\rangle$. Therefore, $\varphi^{-1}\langle u-a, v-b\rangle=\langle t-a\rangle$.

3. Analogously we can ask, does there exist a morphism $f: \mathbf{A}_{\mathbf{C}}^{2} \longrightarrow$ $\mathbf{A}_{\mathbf{C}}^{2}$ such that $f(a, b)=(a b, b)$ on the closed points of $\mathbf{A}_{\mathbf{C}}^{2}$ ?

Define,

$$
\begin{aligned}
\varphi: \mathbf{C}[x, y] & \longrightarrow \mathbf{C}[u, v] \\
p(x, y) & \longmapsto p(u v, v) .
\end{aligned}
$$

Then note that $\varphi(y-b)=v-b$ and $\varphi(x-a b)=u v-a b=$ $u(v-b)+b(u-a)$. Therefore, $\varphi^{-1}\langle u-a, v-b\rangle=\langle x-a b, y-b\rangle$.

Finally, let us remember that a function $f: X \longrightarrow Y$ between topological spaces is said to be dominant if $\overline{f(X)}=Y$, that is, if the image of $f$ is dense in $Y$.

4.4. Remark. Let $\varphi: A \longrightarrow B$ be a ring homomorphism that determines the morphism $f: \operatorname{Spec}(B) \longrightarrow \operatorname{Spec}(A)$, then

$$
f \text { is dominant } \Longleftrightarrow \operatorname{Kernel}(\varphi) \subset \bigcap_{P \in \operatorname{Spec}(A)} P
$$

where

$$
\bigcap_{P \in \operatorname{Spec}(A)} P=N_{A}=\left\{a \in A \mid a^{n}=0 \text { for some } n \in \mathbf{N}\right\}
$$

is called the nilradical of the ring $A$ (see problem $21(\mathrm{v})$, p. 13 in AtiyahMacDonald [1]).

4.5. Example. The homomorphism $\varphi: \mathbf{C}[x, y] \longrightarrow \mathbf{C}[u, v]$ in the example 4.3 3., that determines $f$, is injective. Thus we have $\operatorname{Kernel}(\varphi)=\{0\} \subset$ $N_{\mathbf{C}[x, y]}=\{0\}$. Therefore, $f$ is dominant. 


\section{The notion of dimension}

In this section, we consider the notion of dimension from an algebraic and topological point of view.

- Algebraic

The Krull dimension

Let $A$ be a commutative ring and $\operatorname{Spec}(A)$ the prime spectrum of $A$. Consider all the chains of the form

$$
P_{0} \subset P_{1} \subset \ldots \subset P_{n}
$$

with $P_{i} \in \operatorname{Spec}(A)$. Then the Krull dimension of $A$ is

$$
\operatorname{dim}_{\text {Krull }} A=\max \left\{n \mid P_{0} \subset P_{1} \subset \ldots \subset P_{n} \text { is a chain of } \mathrm{A}\right\} .
$$

The transcendence degree

The concept of dimension also has a close relation with the maximum number of algebraically independent variables in a field. More explicitly, let us consider a finitely generated $\mathbf{K}$-algebra $A$ ( $\mathbf{K}$ is a field), that is, there exists a surjective ring homomorphism $\varphi: \mathbf{K}\left[x_{1}, \ldots, x_{n}\right] \longrightarrow A$.

Note that, if $A$ is an integral domain then $\operatorname{Frac}(A)$, the field of fractions of the ring $A$, is an extension field of $\mathbf{K}$. And we can ask for the maximum number of algebraically independent elements of $\operatorname{Frac}(A)$ over $\mathbf{K}$, that is, the transcendence degree of $\operatorname{Frac}(A)$ over $\mathbf{K}$.

- Topological

Let $X$ be a topological space and $Y \subseteq X$ be a subset of $X$. Consider $Y$ equipped with the natural topology induced from that of $X . Y$ is called $i r$ reducible if $Y$ is not union of two proper closed subsets of $Y$, see Hartshorne [10] and Atiyah-MacDonald [1].

Consider all the chains of the form

$$
Y_{0} \subset Y_{1} \subset \ldots \subset Y_{n}
$$

with $Y_{i} \subset X$ closed irreducible. Then the dimension of $X$ is

$$
\operatorname{dim} X=\max \left\{n \mid Y_{0} \subset Y_{1} \subset \ldots \subset Y_{n} \text { is a chain of } \mathrm{X}\right\} .
$$


5.1. Remark. Note that, if $X$ is a noetherian topological space (see Hartshorne [10], Atiyah-MacDonald [1]), then $X$ admits a decomposition as follows

$$
X=X_{1} \cup \cdots \cup X_{m}
$$

where each $X_{i}$ is an irreducible closed subset of $X$, such that, for all $i \neq j \in$ $\{1, \ldots, m\} X_{i} \not \nsubseteq X_{j}$. Each one of these $X_{i}$ is called an irreducible component of $X$ and in this case it is verified that,

$$
\operatorname{dim} X=\max \left\{\operatorname{dim} X_{i} \mid i \in\{1, \ldots, m\}\right\} .
$$

Thus, in order to compute dimensions of topological spaces, it will be enough to consider the case of irreducible ones.

Let us see the following example.

5.2. Example. Let $X=\operatorname{Spec}\left(\mathbf{C}\left[x_{1}, x_{2}, x_{3}\right] /\left\langle x_{1} x_{2}, x_{1} x_{3}\right\rangle\right) \subset \mathbf{A}_{\mathbf{C}}^{3}$. Note that

$$
\left\langle x_{1} x_{2}, x_{1} x_{3}\right\rangle=\left\langle x_{1}\right\rangle \cap\left\langle x_{2}, x_{3}\right\rangle .
$$

Therefore $X=V\left(x_{1}\right) \cup V\left(x_{2}, x_{3}\right)$. Thus $X$ consists of two irreducible components, namely, the line $V\left(x_{2}, x_{3}\right)$ and the plane $V\left(x_{1}\right)$. And we conclude that $\operatorname{dim} X=2$.

It is important to note that these three approaches to the notion of dimension agree, as the following theorem guarantees.

5.3. Theorem. Let $X=\operatorname{Spec}(A)$ with $A$ a finitely generated $\mathbf{K}$-algebra without divisors of zero then

$$
\operatorname{dim} \operatorname{Spec}(A)=\operatorname{dim}_{\text {Krull }} A=\operatorname{trdeg}_{\mathbf{K}} K(X)
$$

where $K(X)=\operatorname{Frac}(A)$ is called the field of rationals functions of $X$.

Proof. See Lecture 11, Theorem 1, p. 70 in Dolgachev [5]. 


\subsection{Remarks.}

1. Note that, $\operatorname{Spec}(A)$ and $\operatorname{Spec}\left(A / N_{A}\right)$ are isomorphic as topological spaces, hence we have that $\operatorname{dim} \operatorname{Spec}(A)=\operatorname{dim} \operatorname{Spec}\left(A / N_{A}\right)$, where $N_{A}$ is the nilradical of $A$ as in (4.1). That is, in order to compute the dimension of an affine scheme we can assume that its coordinate ring is reduced. More precisely, from remark 5.1 we can assume that its coordinate ring is an integral domain.

2. Let $\mathrm{x} \in \operatorname{Spec}(A)$ be a point in $\operatorname{Spec}(A)$ then $\{\mathrm{x}\}$ can be identified with $\operatorname{Spec}(\kappa(\mathrm{x}))$, where $\kappa(\mathrm{x})=\operatorname{Frac}(A / \mathrm{x})$ is the residual field of x. If $A$ is an integral domain then we have that $\operatorname{Spec}(A)=V(0)$, since 0 is a prime ideal of $A$ and it is contained in all the ideals of $A$. Thus we have that 0 is the generic point of $\operatorname{Spec}(A)$ and in fact it is the only one (see p. 80 in [10]). On the other hand, note that,

$$
\begin{aligned}
& K(\overline{\{0\}})=K(\operatorname{Spec}(A))=\operatorname{Frac}(A), \\
& K(\{0\})=K(\operatorname{Spec}(\kappa(0)))=\kappa(0)=\operatorname{Frac}(A) .
\end{aligned}
$$

Therefore, $K(\operatorname{Spec}(A))=K(\{0\})$.

\section{Preparatory results}

From now on, we will assume that all the rings are finitely generated $\mathbf{K}$ algebras and also integral domains ( $\mathbf{K}$ is a field).

Let $f: X=\operatorname{Spec}(A) \longrightarrow Y=\operatorname{Spec}(B)$ be a morphism of finite type, that is, $A$ is a finitely generated $B$-algebra. Let y $\in Y$ then $\{\mathrm{y}\}$ can be identified with $\operatorname{Spec}(\kappa(\mathrm{y}))$, where $\kappa(\mathrm{y})=\operatorname{Frac}(B / \mathrm{y})$ is the residual field of $\mathrm{y}$.

Having in mind remark 3.2 2. and the definition of Fibered Products (see p. 35 in Harris-Eisenbud [9]), we have the following diagram

$$
\begin{aligned}
& X=\operatorname{Spec}(A) \hookleftarrow f^{-1}(\mathrm{y})=\operatorname{Spec}\left(\kappa(\mathrm{y}) \otimes_{B} A\right) \hookrightarrow f^{-1}(\overline{\{\mathrm{y}\}})=\operatorname{Spec}\left(B / \mathrm{y} \otimes_{B} A\right) \\
& \downarrow f \quad \downarrow \quad \downarrow \\
& Y=\operatorname{Spec}(B) \hookleftarrow \quad\{\mathrm{y}\}=\operatorname{Spec}(\kappa(\mathrm{y})) \quad \hookrightarrow \quad \overline{\{\mathrm{y}\}}=V(\mathrm{y})=\operatorname{Spec}(B / \mathrm{y})
\end{aligned}
$$

In order to determine the dimension of the fiber $f^{-1}(\mathrm{y})$, we need the following two lemmas.

6.1. Lemma. $f^{-1}(\mathrm{y})$ is of finite type over $\{\mathrm{y}\}$. In particular, if $Z \subseteq$ $f^{-1}(\mathrm{y})$ is an irreducible component of $f^{-1}(\mathrm{y})$, then it is verified that, $Z$ is of finite type over $\{\mathrm{y}\}$. 
Proof. We have to show that $R=\kappa(\mathrm{y}) \otimes_{B} A$ ( $R$ is the coordinate ring of $\left.f^{-1}(\mathrm{y})\right)$ is a finitely generated $\kappa(\mathrm{y})$-algebra $(\kappa(\mathrm{y})$ is the coordinate ring of $\{\mathrm{y}\})$. Note that, by hypothesis, $A$ is a finitely generated $B$-algebra, then there exist $b_{1}, \ldots, b_{k} \in A$ such that $A=B\left[b_{1}, \ldots, b_{k}\right]$. Thus we have that,

$$
\kappa(\mathrm{y}) \otimes_{B} A=\kappa(\mathrm{y}) \otimes_{B} B\left[b_{1}, \ldots, b_{k}\right] \cong \kappa(\mathrm{y})\left[b_{1}, \ldots, b_{k}\right] .
$$

In case that $Z \subseteq f^{-1}(\mathrm{y})$ is an irreducible component of $f^{-1}(\mathrm{y})$, then we have that $Z=\operatorname{Spec}(R / P)$, for some minimal prime ideal $P \subset R$.

6.2. Remark. Having in mind exercise 3.20 (b), p. 94 in Hartshorne [10] and using the above lemma, we have that

$$
\operatorname{dim} Z=\operatorname{trdeg}_{\kappa(\mathrm{y})} K(Z)
$$

for any irreducible component $Z$ of $f^{-1}(\mathrm{y})$.

6.3. Lemma. Let $Z \subseteq f^{-1}(y)$ and $Z^{\prime} \subseteq f^{-1}(\overline{\{y\}})$ be irreducible components such that $Z \subseteq Z^{\prime}$. Then we have that $\bar{Z}=Z^{\prime}$ and $K(Z)=K\left(Z^{\prime}\right)$.

Proof. Let $z \in Z$ and $z^{\prime} \in Z^{\prime}$ be their respective generic points. Since we are considering the topology induced on $Z$ and $Z^{\prime}$ from that of $X$, we have that

$$
Z=\overline{\{z\}}^{Z}=\overline{\{z\}} \cap Z \quad \text { and } \quad Z^{\prime}=\overline{\left\{z^{\prime}\right\}} \quad\left(Z^{\prime} \text { is closed in } X\right)
$$

Thus,

$$
\overline{\{z\}}^{Z}=Z=Z^{\prime} \cap Z=\overline{\left\{z^{\prime}\right\}} \cap Z={\overline{\left\{z^{\prime}\right.}}^{Z} .
$$

Therefore, from remark 5.42 . we conclude that $z=z^{\prime}$.

On the other hand, $\{z\} \subset Z \subset Z^{\prime}$. Then we conclude that $\bar{Z}=Z^{\prime}$.

Again from remark 5.42 . we have that

$$
K\left(Z^{\prime}\right)=K\left(\left\{z^{\prime}\right\}\right)=K(\{z\})=K(Z) .
$$




\section{Main result}

Now we will apply Theorem 1.2 and proof our main result.

Theorem. (Fiber dimension theorem) Let $X$ and $Y$ be affine schemes, $f: X \longrightarrow Y$ be a dominant morphism. Then there exists a nonempty open subset $U \subseteq Y$ such that $\operatorname{dimf}^{-1}(\mathrm{y})=r$ for all $\mathrm{y} \in U(r=\operatorname{dim} X-\operatorname{dim} Y)$.

Proof. First of all we choose $U$ as in Theorem 1.2 and we will analyze the following two cases.

Case 1: Let $\mathrm{y} \in Y$ be a closed point such that $\mathrm{y} \in U$.

Let us consider $W=\{\mathrm{y}\}$ and note that $W$ is an irreducible closed subset of $Y$ such that $W \cap U \neq \emptyset$, then any irreducible component $Z$ of the fiber $f^{-1}(\mathrm{y})$ that intersects the inverse image of $U$ has dimension exactly $r$.

Case 2: Let $\mathrm{y} \in Y$ be a non-closed point such that $\mathrm{y} \in U$.

Now let $W^{\prime}=\overline{\{\mathrm{y}\}}$ be the closure of the point $\mathrm{y} \in Y$ and note that $W^{\prime}$ is an irreducible closed subset of $Y$ such that $W^{\prime} \cap U \neq \emptyset$, then any irreducible component $Z^{\prime}$ of $f^{-1}\left(W^{\prime}\right)$ that intersects the inverse image of $U$ has dimension exactly $\operatorname{dim} W^{\prime}+r$. Due to remark 5.41 . we can assume that $W^{\prime}$ is reduced. Thus $K\left(W^{\prime}\right)=K(\{\mathrm{y}\})=\kappa(\mathrm{y})$.

On the other hand, let $Z$ be a component of $f^{-1}(\mathrm{y})$; then there exists a component $Z^{\prime}$ of $f^{-1}\left(W^{\prime}\right)$ such that $Z \subset Z^{\prime}$. Thus from lemma 6.3 we have that $K(Z)=K\left(Z^{\prime}\right)$.

Also from (6.1) we conclude that $\operatorname{dim} Z=\operatorname{trdeg}_{k(\mathrm{y})} K(Z)$. Therefore

$$
\begin{aligned}
\operatorname{dim} Z & =\operatorname{trdeg}_{k(\mathrm{y})} K(Z) \\
& =\operatorname{trdeg}_{k} K(Z)-\operatorname{trdeg}_{k} k(\mathrm{y}) \\
& =\operatorname{trdeg}_{k} K\left(Z^{\prime}\right)-\operatorname{trdeg}_{k} K\left(W^{\prime}\right) \\
& =\operatorname{dim} Z^{\prime}-\operatorname{dim} W^{\prime} \\
& =r .
\end{aligned}
$$

\section{Acknowledgments}

The authors wish to express their gratitude to Francesco Russo (DMIUniversità degli Studi di Catania) for all the suggestions that allowed us to complete the proof in the last section and to the Instituto de Matemática Pura e Aplicada (IMPA), Rio de Janeiro, that provided us the right environment for concluding this work. 


\section{References}

[1] M. F. Atiyah \& I. G. Macdonald, Introduction to Commutative Algebra, Addison-Wesley, Reading, MA, (1969).

[2] D. Avritzer \& I. Vainsencher, Hilb ${ }^{4} \mathbf{P}^{2}$, Lecture Notes in Mathematics, 1436, Springer Verlag, pp. 30-59, (1990).

[3] A. Beauville, Complex Algebraic Surfaces, Astérisque 54, pp. 63$66,(1978)$.

[4] B. Crauder \& R. Miranda, Quantum Cohomology of Rational Surfaces, In: The Moduli Space of Curves, Edited by R. Dijkgraaf, C. Faber, and G. van der Geer. Birhauser Press, Boston, pp. 35-82, (1995).

[5] I. Dolgachev, Introduction to Algebraic Geometry, http://www. math. lsa. umich.edu/ ${ }^{\sim}$ idolga/lecturenotes.html.

[6] D. Eisenbud, Commutative Algebra with a View Toward Algebraic Geometry, Graduate Texts in Math. 150, Springer, New York, (1995).

[7] B. Harbourne, Problems and Progress: A survey on fat points in $\mathbf{P}^{2}$, Queen's papers in Pure and Applied Mathematics, The Curves Seminar at Queen's, vol. 123, (2002).

[8] J. Harris, Algebraic Geometry: A first course, Graduate Texts in Math. 133, Springer, New York, (1995).

[9] J. Harris \& D. Eisenbud, The Geometry of Schemes, Graduate Texts in Math. 197, Springer, New York, (1999).

[10] R. Hartshorne, Algebraic Geometry, Graduate Texts in Math. 52, Springer, New York, (1977).

[11] D. Mumford, The Red Book of Varieties and Schemes, Lecture Notes in Math. 1358, Springer, Berlin, (1988).

[12] M. Reid, Undergraduate Algebraic Geometry, London Mathematical Society Students Texts 12, Cambridge University Press, Cambridge and New York, (1988).

[13] J. Rojas \& I. Vainsencher, Conical Sextuplets, Communications in Algebra, 24(11), pp. 3437-3457, (1996). 
Jacqueline Rojas

UFPB - CCEN - Departamento de Matemática

Campus I - Cidade Universitária

58051-900 - J. Pessoa - PB -

Brasil

e-mail: jacq@mat.ufpb.br

and

Ramón Mendoza

UFPE - CCEN - Departamento de Matemática

Cidade Universitária

50740-540 - Recife - PE -

Brasil

e-mail: ramon@dmat.ufpe.br 